\title{
Variation in Surgical Treatment of Abdominal Aortic Aneurysms With Small Aortic Diameters in the Netherlands
}

\author{
Eleonora G. Karthaus, MD, ${ }^{*} \dagger$ Anco Vahl, MD, PhD, $\ddagger \S L e o n i e ~ R$. van der Werf, MD, $\uparrow$ \\ Bernard H. P. Elsman, MD, PhD, $\|$ Joost A. Van Herwaarden, MD, PhD, ${ }^{* *}$ \\ Michel W. J. M. Wouters, MD, PhD, † and Jaap F. Hamming, MD, PhD*
}

\begin{abstract}
Objective: To evaluate reasons to deviate from aneurysm diameter thresholds, and focus on the difference in how Dutch vascular surgical units (VSUs) perceive their deviation and their actual deviation.

Background: Guidelines recommend surgical treatment for asymptomatic abdominal aortic aneurysms (AAAs) with a diameter of at least $55 \mathrm{~mm}$ for men and $50 \mathrm{~mm}$ for women. We evaluate reasons to deviate from these guidelines, and focus on the difference in how Dutch vascular surgical units (VSUs) perceive their deviation and their actual deviation.

Methods: All patients undergoing elective AAA repair between 2013 and 2016 registered in the Dutch Surgical Aneurysm Audit (DSAA) were included. Surgery at diameters of $<55 \mathrm{~mm}$ for men and $<50 \mathrm{~mm}$ for women were considered guideline deviations. National deviation and hospital variation in deviation were evaluated over time. Questionnaires were distributed among all Dutch VSUs, inquiring for acceptable reasons for guideline deviation. VSUs were asked to estimate the guideline deviation percentage in their hospital which was then compared with their DSAA percentage.

Results: In all, 9039 patients were included. In 15\%, we found guideline deviation, varying from $2 \%$ to $40 \%$ between VSUs. Over time, 21 VSUs were identified with a lower percentage of deviation than the national mean each year and 8 VSUs with a higher percentage. 44/60 VSUs completed the questionnaire. Most commonly reported reasons to deviate were concomitant large iliac diameter (91\%) and saccular aneurysm (82\%). The majority of the VSUs (77\%) estimated their guideline deviation to be $<5 \%$. Eleven VSUs (25\%) estimated their deviation concordant with their DSAA percentage, but $75 \%$ of VSUs underestimated their deviation.

Conclusions: Dutch VSUs regularly deviate from the guidelines regarding aneurysm diameter, with variation between VSUs. Consensus exists amongst VSUs on acceptable reasons for guideline deviations; however, the majority underestimates their actual deviation percentage.
\end{abstract}

Keywords: diameter threshold, elective abdominal aortic aneurysm, guideline deviation, small abdominal aortic aneurysm

(Ann Surg 2020;271:781-789)

From the *Department of Surgery, Leiden University Medical Center, Leiden, The Netherlands; $\nmid$ Dutch Institute for Clinical Auditing, Leiden, The Netherlands; $\ddagger$ Department of Surgery, OLVG, Amsterdam, The Netherlands; §Department of Clinical Epidemiology, OLVG, Amsterdam, The Netherlands; †Department of Surgery, Erasmus Medical Center, Rotterdam, The Netherlands; ||Department of Surgery, Deventer Hospital, Deventer, The Netherlands; and ${ }^{* *}$ Department of Vascular Surgery, University Medical Center Utrecht, Utrecht, The Netherlands.

Funding: This research received no specific funding.

The authors declare that they have no conflicts of interest

Supplemental digital content is available for this article. Direct URL citations appear in the printed text and are provided in the HTML and PDF versions of this article on the journal's Web site (www.annalsofsurgery.com).

Reprints: Eleonora G. Karthaus, Department of Surgery (Zone K6-R), Leiden University Medical Center, Albinusdreef 2, 2333 ZA Leiden, the Netherlands. E-mail: n.karthaus@dica.nl,e.g.karthaus@lumc.nl.

Copyright (C) 2020 Wolters Kluwer Health, Inc. All rights reserved.

ISSN: 0003-4932/20/27104-0781

DOI: $10.1097 /$ SLA.0000000000003050
$\mathrm{T}^{\mathrm{h}}$ he indication for elective surgical treatment in patients with an asymptomatic abdominal aortic aneurysm (AAA) depends on multiple factors, of which the diameter of the aneurysm is the most important one, as the risk of rupture increases with the diameter of the aneurysm. ${ }^{1}$ International guidelines recommend surgical treatment in patients with an asymptomatic AAA, with a diameter of $55 \mathrm{~mm}$ or more in males and $50 \mathrm{~mm}$ or more in females. ${ }^{2,3}$ These diameter thresholds for intervention have been studied extensively, and early intervention in asymptomatic patients with a small AAA ( $<55 \mathrm{~mm}$ in males, $<50 \mathrm{~mm}$ in females) has not proven to be beneficial compared with watchful waiting. ${ }^{4-8}$

Since 2013, all patients undergoing aortic aneurysm surgery in the Netherlands are registered in a nationwide audit — the Dutch Surgical Aneurysm Audit (DSAA). ${ }^{9}$ This audit reported previously that $17 \%$ of all patients undergoing elective aneurysm surgery is operated with a smaller diameter than recommended in the guidelines, with variation between hospitals. ${ }^{10}$ Other studies have also confirmed variation in practice regarding the aneurysm diameter, nationally and internationally. ${ }^{11-13}$ There are reasons why surgeons could decide to deviate from this guideline, for example, a saccular-shaped aneurysm, a large iliac component, rapid growth, and so on. ${ }^{14}$ However, unnecessarily, large variation in clinical practice is undesirable, because it can result in unnecessary adverse outcomes for patients ${ }^{13}$ and will lead to unnecessary costs. ${ }^{15,16}$ To minimize differences in practice, to improve quality of care, and to use health care more efficiently, it is important to have more insight into the reasons for this variation in clinical practice.

The aim of this study is first to evaluate patient and disease characteristics associated with performing surgical therapy on patients with a smaller aortic diameter than recommended in the guideline and secondly to investigate reasons to deviate from this guideline with a focus on how often Dutch vascular surgical units (VSUs) think they deviate from the guidelines and actually do.

\section{METHODS}

This study consists of the following 3 parts:

1. Analysis of national data from the DSAA

2. Survey questionnaire among Dutch Vascular Surgeons

3. Comparison of the outcomes of the survey questionnaire and DSAA data

\section{Part 1: Analysis of National Data from the Netherlands}

\section{Data Source and Patient Selection}

The dataset was derived from the DSAA. This compulsory nationwide audit was initiated in 2013 and prospectively registers all patients undergoing surgery for an aortic aneurysm or dissection. Data are registered via a web-based survey or provided by the hospitals as a batch data file. All patients with a juxta or infrarenal AAA undergoing primary elective surgery between January, 2013 
and December, 2016 were included. All patients with symptomatic or ruptured AAAs, isolated iliac artery aneurysms, thoracic aortic aneurysms or/and dissections, undefined aneurysms, and patients undergoing revision surgery were excluded. Additionally, patients operated in hospitals that stopped performing aneurysm surgery after the first year of the study period were also excluded.

\section{Aneurysm Diameter}

In the survey of the DSAA, the largest measured aortic aneurysm diameter, anterior-posterior measured with ultrasound or computed tomography angiography (CTA) and extracted from the radiology report, is registered. The diameter thresholds for surgical treatment in asymptomatic AAAs according to the Dutch national guideline are used: $55 \mathrm{~mm}$ or more for males, $50 \mathrm{~mm}$ or more for females. We have made a distinction between 'any deviations' from this guideline (diameter $<55 \mathrm{~mm}$ in males, diameter $<50 \mathrm{~mm}$ in females), 'small deviations' (diameter from 50 to $54 \mathrm{~mm}$ in males, and from 45 to $49 \mathrm{~mm}$ in females), and 'large deviations' (diameter $<50 \mathrm{~mm}$ in males, diameter $<45 \mathrm{~mm}$ in females).

\section{Dutch Healthcare Policies Regarding Elective AAA Surgery}

For the treatment of elective AAA, there is an annual minimum volume standard of 20 elective AAA repairs per hospital in the Netherlands. This minimum volume standard is monitored with the use of DSAA data. All patients undergoing elective AAA surgery are preoperatively discussed in a multidisciplinary team or vascular meeting. This is also a quality indicator that is monitored in the DSAA.

\section{Analysis}

Using descriptive analysis ( $t$ test and chi-square test), patient, disease, and treatment characteristics were compared between 2 separate groups: all patients treated according to the national guidelines and all patients in whom the guidelines were deviated.

Patient, treatment, and hospital characteristics independently associated with any deviation from the guidelines were evaluated using a multivariable logistic regression analysis, with $P$ value of 0.05 using an enter model. Covariables used in this multivariable logistic regression analysis were: sex, age, pulmonary state, cardiac state, results of last preoperative electrocardiogram, malignancy, preoperative hemoglobin and creatinine, type of surgical procedure, and hospital volume.

Additionally, variation in surgical treatment of small aneurysms (diameter $<55 \mathrm{~mm}$ in males, diameter $<50 \mathrm{~mm}$ in females) between hospitals was evaluated over time, by comparison of the percentage of deviation from the guideline per hospital over the years 2013 to 2016.

All statistical analyses were performed using SPSS statistical software (version 24; IBM Corp, Armonk, NY).

\section{Part 2: Survey Questionnaire Among Dutch Vascular Surgeons}

To obtain insight into the reasons why vascular surgeons decide to operate patients with a small AAA diameter, an online survey questionnaire was distributed among VSUs in the 60 hospitals that perform AAA surgery in the Netherlands. The contact person for the DSAA of each VSU-Chief of the Department of Vascular Surgery-was contacted to fill in the questionnaire for his/her VSU. The survey consisted of 14 questions (Appendix 1, http:// links.lww.com/SLA/B513). In the first section, units were asked to estimate how often they perform surgery on patients with a small aortic aneurysm diameter in 2 multiple choice questions.
Subsequently, they were asked what they thought to be acceptable reasons to deviate from the guideline, in which multiple reasons were proposed. Finally, they were asked to estimate to what extent these 11 reasons were applicable to or did occur in their hospital, by using a Likert scale. To compare the results of the questionnaire with the DSAA data, units were asked to report the name of their hospital, making the questionnaire not anonymous.

Descriptive analyses were used to evaluate outcomes.

\section{Part 3: Comparison of the Outcomes of the Survey Questionnaire and DSAA Data}

Results of de survey questionnaire were compared with the DSAA data on hospital level. Discrepancies between the estimated percentage of guideline deviation by the VSUs and their actual practice were evaluated, and also the differences in reasons to deviate from guidelines between hospitals with high and low guideline adherence.

\section{RESULTS}

\section{Part 1: Analysis of National Data From the Netherlands}

Between January, 2013 and December, 2016, in all, 10,186 patients underwent elective aneurysm surgery in the Netherlands. After exclusion of 546 patients with an isolated iliac aneurysm, 212 with a (concomitant) thoracic aneurysm/dissection, 209 with an undefined aneurysm, 166 with revision surgery, and 14 patients operated in hospitals that stopped performing AAA surgery, in all, 9039 patients were included for analysis. Out of these patients, $15 \%$ (1324 patients) had a smaller abdominal aortic diameter than in which surgical treatment is recommended by the national guideline, $16 \%$ of all male patients and $9.0 \%$ of all female patients. In $11 \%$ (969), this concerned a small deviation from the guideline and in $3.9 \%$ (355) a large deviation.

Compared with the group of patients treated according to the guideline, there were more male patients in the group which was deviated from the guideline $(91 \%$ vs $85 \% ; P<0.001)$ and this group was on average 3 years younger (mean 70.9 SD 8.0 vs 73.5 SD $7.5 ; P$ $<0.001$ ). Additionally, pulmonary state, cardiac state, preoperative ECG, malignancies, preoperative hemoglobin, and type of surgical procedure were unequally distributed between the 2 groups (Table 1).

\section{Characteristics Associated With Deviation From the Guideline}

Characteristics independently associated with deviation from the guideline were: male sex [odds ratio (OR) 1.709, 95\% confidence interval (CI) 1.386-2.109] and treatment with endovascular aneurysm repair (EVAR) (OR 1.432, 95\% CI 1.232-1.664) (Table 2). Characteristics with a low OR for deviation from the guideline were: age (OR $0.958,95 \%$ CI $0.950-0.966$, per additional year), peripheral edema (OR 0.644, 95\% CI 0.510-0.864), current malignancy (OR $0.560,95 \%$ CI $0.399-0.786$ ), and hospital volume (OR 0.998, 95\% CI 0.997-0.999, per additional procedure).

\section{Hospital Variation}

Between hospitals, the percentage deviations from the guideline varied between $2 \%$ and $40 \%$ (median 13\%) (0\%-33\% small deviations; $0 \%-17 \%$ large deviations) (Fig. 1). When the variation in surgical treatment of small AAAs was evaluated over time, 21 hospitals could be identified with a lower percentage of deviations than the national mean (15\%) of deviations every year (Appendix 2, http://links.lww.com/SLA/B513). Respectively, 12, 6, and 14 hospitals had higher percentage deviations than the national mean of 
TABLE 1. Comparison of Patient Characteristics Between Patients With Guideline Adherence and Guideline Deviation

\begin{tabular}{|c|c|c|c|c|c|}
\hline & Gui & ence & Gu & ion & \\
\hline & $\begin{array}{r}\text { An } \\
\text { (Male } \\
\end{array}$ & $\begin{array}{l}\text { ter } \\
\text { males }\end{array}$ & $\begin{array}{r}\text { Anc } \\
\text { (Male }\end{array}$ & $\begin{array}{l}\text { ter } \\
\text { males }\end{array}$ & \\
\hline Number of patients & 7715 & $85 \%$ & 1324 & $15 \%$ & \\
\hline Age (mean, yrs) & & & & & $<0.001$ \\
\hline Sex & & & & & $<0.001$ \\
\hline Year of surgery & & & & & 0.009 \\
\hline 2013 & 1636 & $21 \%$ & 332 & $25 \%$ & \\
\hline 2014 & 2090 & $27 \%$ & 361 & $27 \%$ & \\
\hline 2015 & 1964 & $26 \%$ & 318 & $24 \%$ & \\
\hline 2016 & 2025 & $26 \%$ & 313 & $24 \%$ & \\
\hline Cardiac state & & & & & $<0.001$ \\
\hline No abnormalities & 3511 & $46 \%$ & 670 & $51 \%$ & \\
\hline Pulmonary state & & & & & 0.044 \\
\hline No dyspnea & 5633 & $73 \%$ & 1015 & $77 \%$ & \\
\hline Dyspnea & 1655 & $22 \%$ & 242 & $18 \%$ & \\
\hline Severe dyspnea & 314 & $4.1 \%$ & 51 & $3.9 \%$ & \\
\hline Unknown & 113 & $1.5 \%$ & 16 & $1.2 \%$ & \\
\hline Malignancy & & & & & 0.002 \\
\hline None & 6217 & $81 \%$ & 1102 & $83 \%$ & \\
\hline Current & 392 & $5.1 \%$ & 39 & $2.9 \%$ & \\
\hline History of malignancy & 1106 & $14 \%$ & 183 & $14 \%$ & \\
\hline Last preoperative ECG & & & & & 0.021 \\
\hline No abnormalities & 4235 & $55 \%$ & 781 & $59 \%$ & \\
\hline Abnormalities & 2731 & $35 \%$ & 429 & $32 \%$ & \\
\hline No ECG performed/unknown ECG & 749 & $9.7 \%$ & 114 & $8.6 \%$ & \\
\hline Hart rate (mean, bpm) & & & & & 0.174 \\
\hline Normal potassium $(3.5-5.0 \mathrm{mmol} / \mathrm{L})$ & 7256 & $94 \%$ & 1232 & $93 \%$ & \\
\hline Hypo/hyperpotassemia & 459 & $5.9 \%$ & 92 & $6.9 \%$ & \\
\hline Treatment & & & & & 0.002 \\
\hline OSR & 1808 & $23 \%$ & 258 & $20 \%$ & \\
\hline EVAR & 5907 & $77 \%$ & 1066 & $81 \%$ & \\
\hline
\end{tabular}

deviations in 1, 2, or 3 years. Finally, 7 hospitals could be identified that had a higher percentage of deviations than the national mean in every year.

\section{Part 2: Survey Questionnaire Among Dutch Vascular Surgical Teams}

In all, 44 (out of 60) VSUs completed the online survey questionnaire ( $73 \%$ response rate). The majority of the units $(\mathrm{n}=$ $34,77 \%$ ) estimated to deviate from the guideline in less than $5 \%$ of their patients. The remaining $9(21 \%)$ and $1(2 \%)$ estimated to deviate from the guidelines in respectively $5 \%$ to $15 \%$ and $>15 \%$ of their patients. Additionally, $42(95.5 \%)$ and $2(4.5 \%)$ units answered that they perform surgery on patients with an aneurysm of more $5 \mathrm{~mm}$ smaller than the recommended threshold (large deviation) in, respectively, $<5 \%$ and $5 \%$ to $15 \%$ of their patient.
Acceptable reasons mentioned to deviate from the guideline were aorto-iliac aneurysm with large iliac diameter $(n=40,91 \%)$, saccular aortic aneurysm $(\mathrm{n}=36,82 \%)$, rapid aneurysm growth $(\mathrm{n}=$ $35,80 \%$ ), and a chronic painful aneurysm (nonacute mild abdominal pain during physical examination) $(\mathrm{n}=27,61 \%)$ (Appendix 3, http:// links.lww.com/SLA/B513). Other suggested reasons were patients desire to undergo aneurysm surgery $(\mathrm{n}=15,34 \%)$, a connective tissue disorder $(\mathrm{n}=10,23 \%)$, younger age of the patient $(\mathrm{n}=6$, $14 \%)$, a positive family history for aortic aneurysm rupture $(\mathrm{n}=5$, $11 \%$ ), afraid that treatment with EVAR would not be possible when the aneurysm would grow further $(\mathrm{n}=1,2 \%)$, other reasons $(\mathrm{n}=3$, $7 \%$ ), and no good reasons $(\mathrm{n}=0,0 \%)$. The reasons to deviate from the guideline that were reported to in fact occur in their own practice were (Appendix 4, http://links.lww.com/SLA/B513): 'concomitant large iliac aneurysm' (regularly 39\%, often 39\%), 'saccular 
TABLE 2. Patient and Hospital Characteristics Independently Associated With Deviation Guideline

\begin{tabular}{|c|c|c|}
\hline & \multicolumn{2}{|c|}{ Deviation From the Guideline } \\
\hline & Odds Ratio & $95 \%$ CI \\
\hline Number of patients & \multicolumn{2}{|c|}{9039} \\
\hline Age (mean, yrs) & 0.958 & $0.950-0.966$ \\
\hline \multicolumn{3}{|l|}{ Sex } \\
\hline Female & Ref. & \\
\hline Male & 1.709 & $1.386-2.109$ \\
\hline \multicolumn{3}{|l|}{ Pulmonary state } \\
\hline No dyspnea & Ref. & \\
\hline Dyspnea & 0.895 & $0.767-1.045$ \\
\hline Severe dyspnea & 1.017 & $0.746-1.386$ \\
\hline Unknown & 0.870 & $0.508-1.491$ \\
\hline \multicolumn{3}{|l|}{ Cardiac state } \\
\hline No abnormalities & Ref. & \\
\hline Peripheral edema & 0.664 & $0.510-0.864$ \\
\hline Raised central venous pressure & 1.042 & $0.647-1.679$ \\
\hline Antihypertensive medication & 0.899 & $0.789-1.023$ \\
\hline Unknown & 0.988 & $0.724-1.348$ \\
\hline \multicolumn{3}{|l|}{ Last preoperative ECG } \\
\hline No abnormalities & Ref. & \\
\hline Abnormalities & 0.994 & $0.868-1.138$ \\
\hline No ECG performed & 0.862 & $0.692-1.074$ \\
\hline \multicolumn{3}{|l|}{ Malignancy } \\
\hline None & Ref. & \\
\hline Current & 0.560 & $0.399-0.786$ \\
\hline History of malignancy & 1.024 & $0.861-1.216$ \\
\hline \multicolumn{3}{|l|}{ Preoperative laboratory results } \\
\hline \multicolumn{3}{|l|}{ Hemoglobin $(\mathrm{mmol} / \mathrm{L})$} \\
\hline$<7.5$ & Ref. & \\
\hline $7.5-8.5$ & 1.096 & $0.869-1.382$ \\
\hline $8.6-9.5$ & 1.112 & $0.893-1.386$ \\
\hline$>9.5$ & 1.136 & $0.896-1.441$ \\
\hline \multicolumn{3}{|l|}{ Creatinine $(\mathrm{mmol} / \mathrm{L})$} \\
\hline$<80$ & Ref. & \\
\hline $80-100$ & 1.062 & $0.916-1.230$ \\
\hline $101-120$ & 1.043 & $0.867-1.255$ \\
\hline$>120$ & 0.994 & $0.814-1.214$ \\
\hline \multicolumn{3}{|l|}{ Treatment } \\
\hline OSR & Ref. & \\
\hline EVAR & 1.432 & $1.232-1.664$ \\
\hline Hospital volume $2013-2016^{*}$ & 0.998 & $0.997-0.999$ \\
\hline
\end{tabular}

*Volume of elective AAA repairs between 2013 and 2016.

aneurysm' (regularly 39\%, often 30\%), and 'rapid aneurysm growth' (regularly $32 \%$, often $25 \%$ ). 'Space on the operating room schedule' and 'achieving volume standard' were never $(0,0 \%)$ reported. 'Afraid that EVAR would not be possible when the aneurysm grows,' 'young age of the patient,' and 'positive family history' were answered to never occur in, respectively, 93\%, 61\%, and $57 \%$ of the units.

\section{Part 3: Comparison of Survey Questionnaire and DSAA Data}

The percentage of deviations from the guideline per vascular surgical unit as registered in the DSAA (Fig. 2, left column) were compared in the survey with the estimated percentage of deviations per unit (Fig. 2, right column). Eleven units had an estimated percentage concordant (green) to their actual practice registered in the DSAA and 33 had not (red). Of the 33 units with nonconcordant estimations, 11 units estimated to deviate from the guideline in $<5 \%$ of the patients while doing that in $>15 \%$. The percentage of large deviations from the guideline per unit as registered in the DSAA compared with the estimated percentage deviations per unit is given in Figure 3. There were 31 units with concordant estimations of large deviations and 13 with nonconcordant estimations. Not responding to the survey (hospitals in gray) does not seem to be associated with higher percentage deviations from the guideline. Differences in patient and hospital characteristics between units that did and did not respond to the survey are shown in Table 3. In the group of nonresponders, there were more high-volume hospitals, and an EVAR procedure was more common.

\section{DISCUSSION}

Dutch VSUs regularly decide to deviate from the guideline regarding aneurysm diameter. Male sex, young age, absence of peripheral edema and current malignancy, treatment with EVAR, and lower hospital volume are factors that are independently associated with performing elective aneurysm repair in patients with a smaller aneurysm diameter than recommended in the guidelines. Guideline deviation varied considerably between units, both for small and large deviations. When the variation in surgical treatment of small AAAs was evaluated over time, units that rarely deviated from the guideline could be identified, and also units that structurally did. Among Dutch VSUs, there is agreement on acceptable reasons to perform elective surgery on patients with a small aortic aneurysm. However, there is considerable variation in the extent to which these reasons occur in actual practice. The estimated percentage of guideline deviations of each unit was often nonconcordant and much lower than the actual practice as registered in the DSAA.

Since the publication of a retrospective review about the incidence of AAA and AAA rupture in nonspecific autopsies, the maximum aneurysm diameter is generally regarded as an important measure of risk for rupture. ${ }^{17}$ International guidelines recommend an aneurysm diameter threshold for elective aneurysm repair of $>55 \mathrm{~mm}$ in males and $>50 \mathrm{~mm}$ in females, based on the balance between the risk of aneurysm rupture and postoperative mortality in elective aneurysm repair. ${ }^{2,3,18}$

Two large randomized controlled trails - the United Kingdom small aneurysm trial (UKSAT) and Aneurysm Detection and Management (ADAM) trail- have evaluated potential benefit of elective aneurysm repair in asymptomatic patients with a diameter between 40 and $54 \mathrm{~mm}$, compared with watchful waiting. ${ }^{4,8}$ In both trials, the postoperative mortality was significantly higher than the rupture rate. Therefore, early intervention is not beneficial. With the advent of EVAR, postoperative mortality in elective aneurysm surgery has strongly decreased. However, more recent studies comparing early EVAR and surveillance have again not shown a mortality benefit for early intervention. ${ }^{5,6,14}$ Therefore, the current diameter thresholds for intervention in patients with asymptomatic aortic aneurysms have not changed. ${ }^{2,3}$

Nevertheless, this study shows that in reality Dutch VSUs regularly decide to perform surgery on patients with smaller aneurysm diameters than the thresholds, with a wide variation between units. Generally, a saccular shape of the aneurysm or an AAA with a large iliac aneurysm component is accepted for early surgical treatment, but high level of evidence is lacking. ${ }^{2,3}$ Moreover, it is suggested that patients with rapid expansion of a small aortic aneurysm may benefit from early repair. ${ }^{2,19,20}$ Patients with connective tissue diseases have an increased risk to develop aortic pathology and therefore it is understandable to perform early intervention on these patients. ${ }^{21,22}$ However, an isolated AAA is rare in patients with connective tissue diseases and therefore it does not seem to be a good reason to deviate from the guideline. ${ }^{23,24}$ The benefit of early intervention for other reasons as young age and positive family history have not been demonstrated or investigated. ${ }^{3}$

Except for connective tissue diseases, mostly treated in centers of expertise, it is plausible that patient and aneurysm characteristics, 


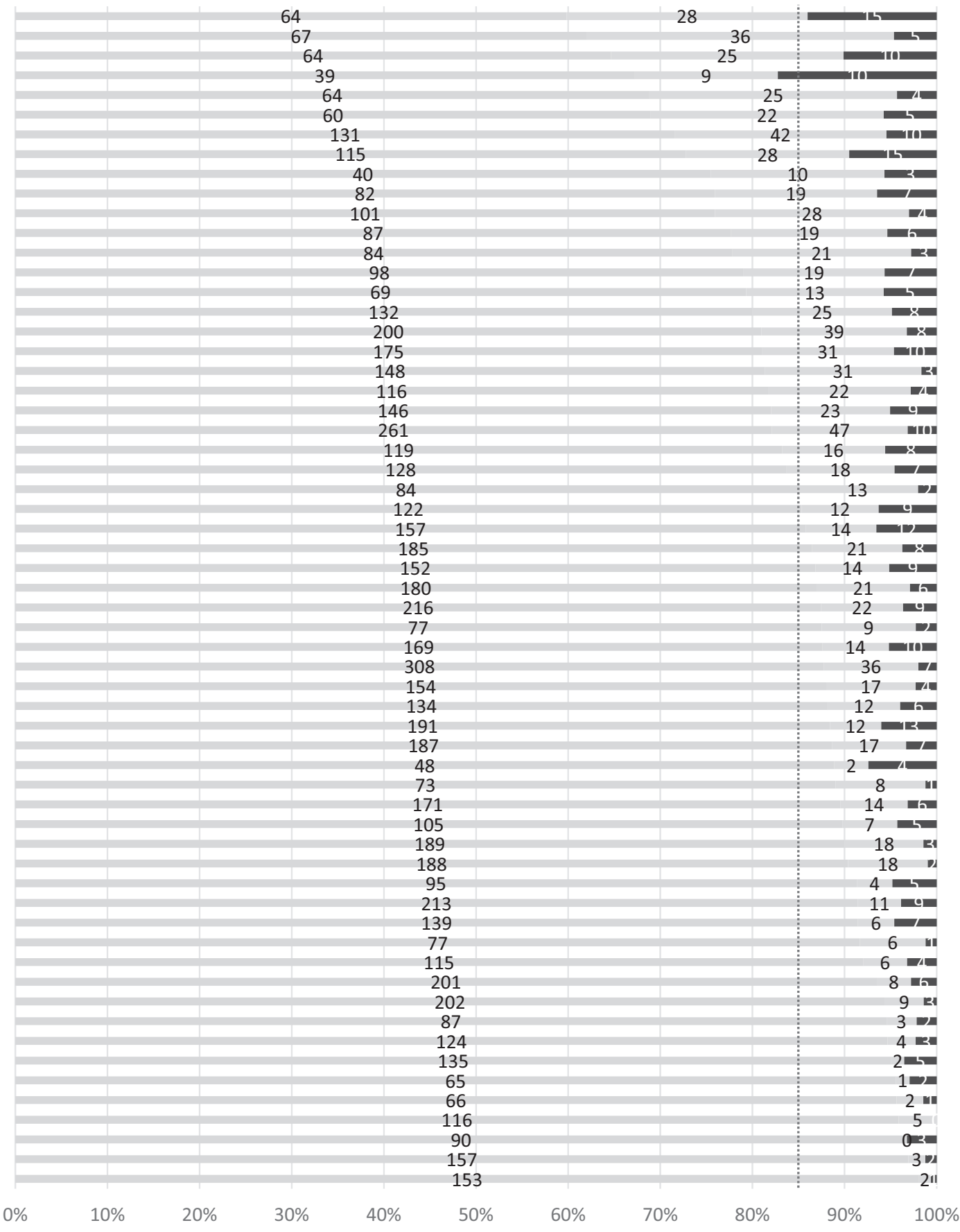

Guideline adherence $\square$ Deviated from guideline $\quad$ Deviated from guideline with $>5 \mathrm{~mm} \quad$..... National mean of deviation

FIGURE 1. Percentage deviations from guideline per vascular surgical unit. 


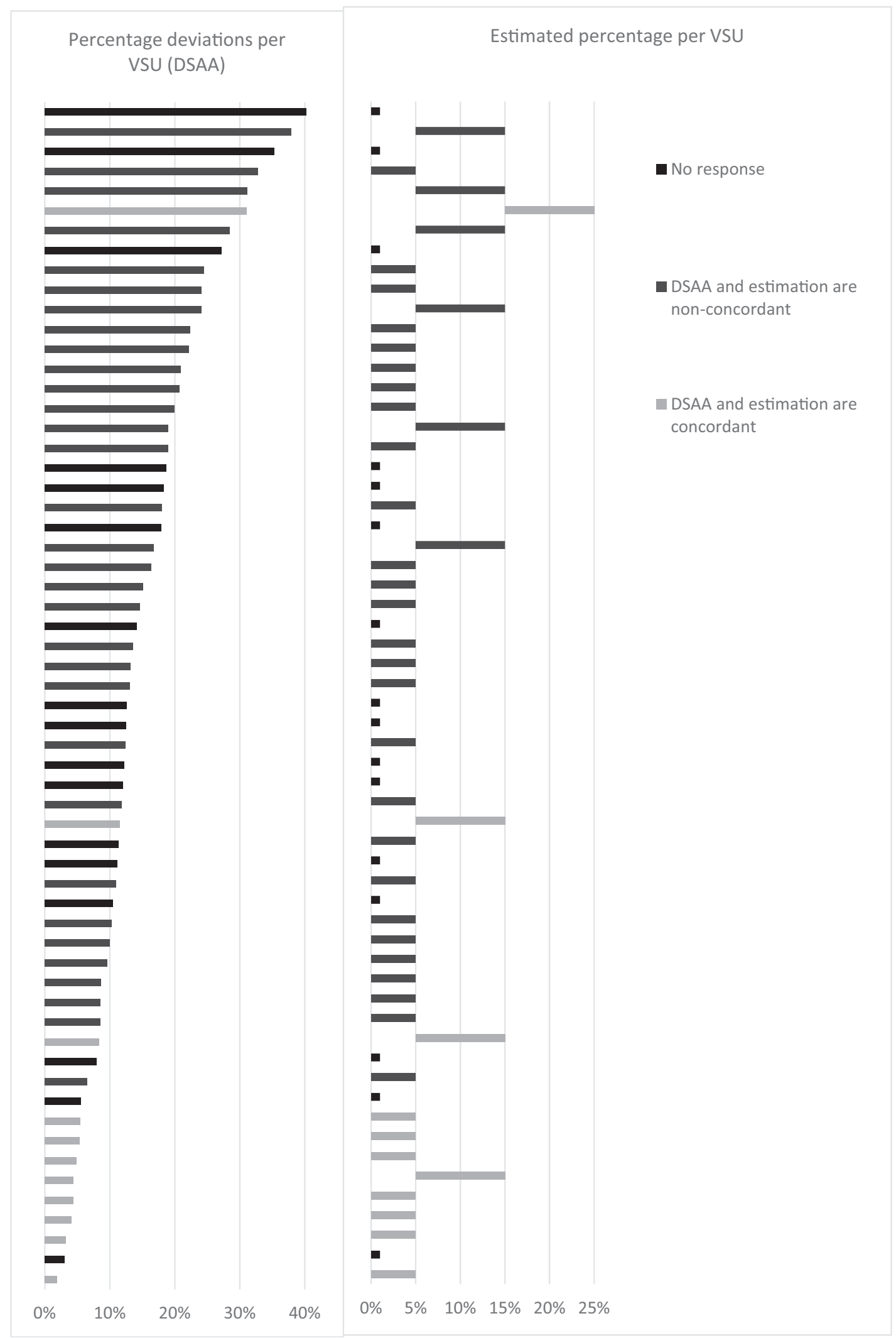

FIGURE 2. Comparison of actual percentage deviations and estimated percentage per vascular surgical unit. 


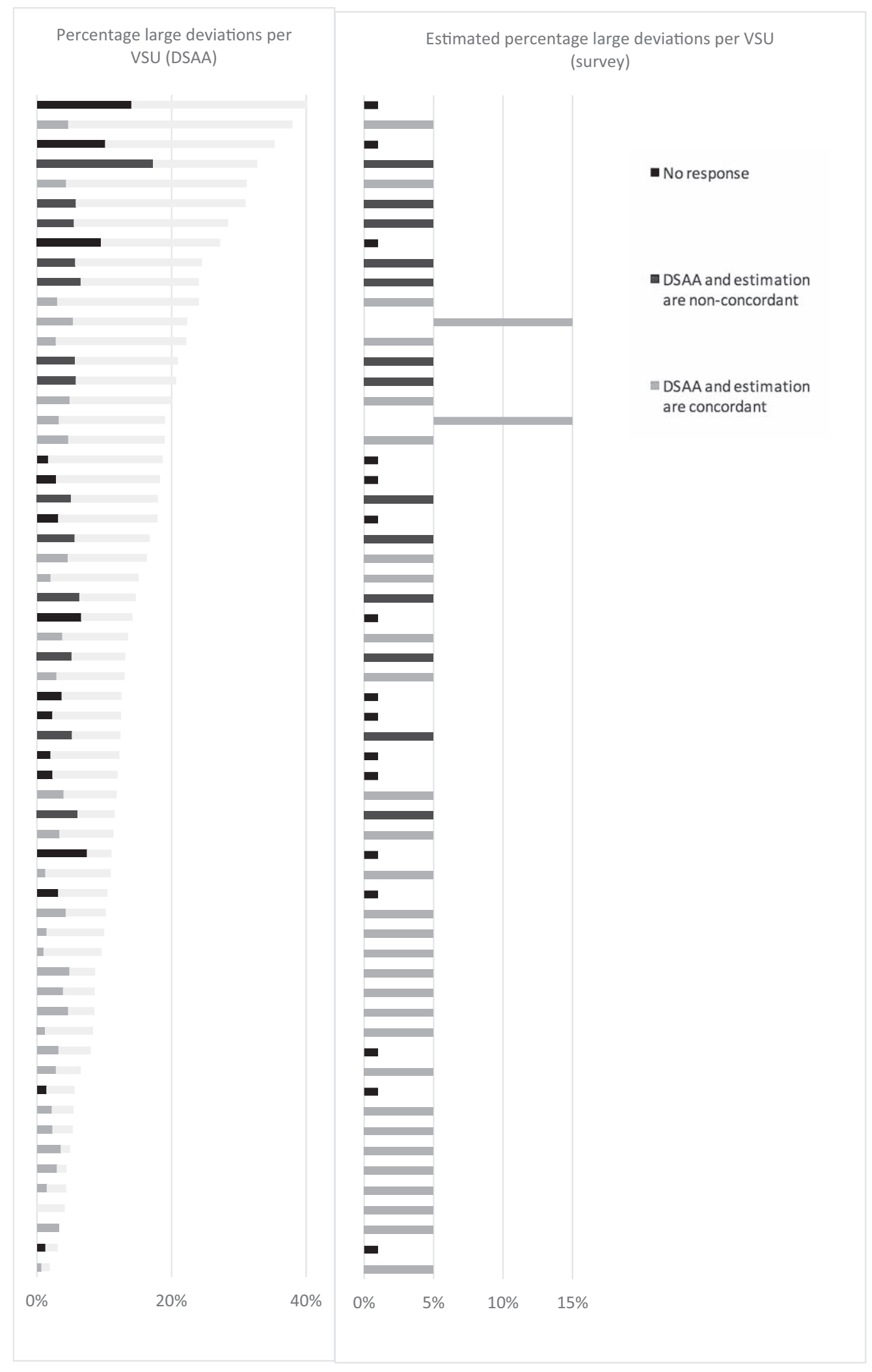

FIGURE 3. Comparison of actual percentage large deviations and estimated percentage per vascular surgical unit. 
TABLE 3. Differences in Patient and Hospital Characteristics Between VSUs That Responded and Did Not Respond to the Questionnaire

\begin{tabular}{|c|c|c|c|c|c|}
\hline & \multicolumn{2}{|c|}{ Units That Responded } & \multicolumn{2}{|c|}{ Units That Did Not Respond } & \\
\hline & $\mathbf{N}$ & $\%$ & $\mathbf{N}$ & $\%$ & \\
\hline Number of patients & 6243 & $69 \%$ & 2796 & $31 \%$ & \\
\hline Age (mean, yrs) & \multicolumn{2}{|c|}{ 73.1 SD 7.7} & \multicolumn{2}{|r|}{ 73.2 SD 7.2} & 0.519 \\
\hline Male & 5315 & $85 \%$ & 2424 & $87 \%$ & \\
\hline Female & 928 & $15 \%$ & 372 & $13 \%$ & \\
\hline Treatment & & & & & 0.000 \\
\hline$<100$ & 965 & $16 \%$ & 241 & $9 \%$ & \\
\hline $100-150$ & 1594 & $26 \%$ & 374 & $13 \%$ & \\
\hline $150-200$ & 1507 & $24 \%$ & 1051 & $38 \%$ & \\
\hline$>200$ & 2177 & $35 \%$ & 1130 & $40 \%$ & \\
\hline Guideline adherence & & & & & 0.387 \\
\hline Guideline adherence & 5342 & $86 \%$ & 2373 & $85 \%$ & \\
\hline
\end{tabular}

*Volume of elective AAA repairs between 2013 and 2016.

and the occurrence of reasons to deviate from the guideline are about equally distributed among hospitals. However, the questionnaire did show some variation between vascular units in how often these reasons resulted in guideline deviation. Indications as saccular aneurysm or aorto-iliac aneurysms with a large iliac component are relatively uncommon; nevertheless in this study, of all reasons, they were most frequently reported as 'regularly' of 'often' occurring. ${ }^{25,26}$

Male sex and treatment with EVAR were independently associated with deviation from the guideline, whereas increasing age, peripheral edema, current malignancy, and high hospital volume were independently associated with adherence. These characteristics do not necessarily correspond to the generally accepted indications, as the mean age of patients with a saccular aneurysm or aorto-iliac aneurysm with large iliac component is comparable with the mean age of aneurysm patients or even higher. ${ }^{25}$ Additionally, as female sex is associated with a higher risk of rupture, it would be expected that guideline deviation would happen more often in female patients than in males. ${ }^{27,28}$ This suggests that in relatively young males with little to no comorbidities that are eligible for treatment with EVAR, surgical treatment is more often chosen for smaller aneurysm diameters than recommended in the guideline. Another interesting finding is that hospitals with lower volumes performed surgery more often on patients with smaller diameter than hospitals with higher volumes. Apart from achieving volume standards, financial incentives may also play a role in deviating from the guideline. ${ }^{11}$

When evaluating deviation from the guideline over time between units, units with a lower percentage of deviations than the national mean could be identified, and also units with a higher percentage of deviations than the national mean in every year. Apparently, there is a certain consistency in the behavior of VSUs to perform surgery or not on patients with a smaller aortic diameter. Remarkably, VSUs with a higher total percentage of guideline deviations more often had an estimation nonconcordant with their actual practice than VSUs with a lower total percentage of deviations. It seems that VSUs that frequently deviate from the guideline are apparently not aware that they are doing this.

This study has several limitations. To evaluate the national performance and difference between surgical teams regarding surgical treatment for small AAAs, it would have been useful to know the exact reason to deviate from the guideline for each patient. Unfortunately, this information was not captured in the DSAA. By combining information about the incidence of deviation from the DSAA and information about the reasons and occurrence of these reasons from our questionnaire, we have tried to approach the proportion of different reason per vascular surgical unit to get more insight into variation in practice between units.

Secondly, the measurement of aneurysm diameter registered in the DSAA is not standardized. For the surveillance of patients with an asymptomatic AAA ultrasound is the imaging modality of preference. However, it may be possible that diameters measured with CTA are registered as well. It is known that a diameter of an aneurysm is often larger when measured with CTA compared with ultrasound. This could result in an underestimation of the actual percentage of guideline deviation. ${ }^{3}$ As we mainly focus on the decision-making after the measurement, this problem probably is not relevant.

Lastly, although a $73 \%$ response rate on a national questionnaire is quite good, we were not able to provide information on the reported reasons of all Dutch VSUs. However, as the percentage of guideline deviation was not associated with not responding to the questionnaire and not responding appears to be coincidental, we consider the sample representative.

Guideline deviations happen often and extensively, and most hospitals that frequently deviate from the guideline do not seem to be aware of the fact that they are doing so. Therefore, providing good feedback information to vascular units is important for their process of quality-of-care improvement. The DSAA has an online portal in which vascular units can review their performance on multiple domains and compare this to other units. From January, 2018, the percentage of guideline deviation regarding aneurysm diameter in elective AAA patients, compared with the national mean and the percentage of all other vascular units, will be fed back to the units. Consequently, teams will be more aware how they perform, and hopefully variation, in practice, will decrease. ${ }^{29}$

\section{CONCLUSIONS}

In conclusion, deviations from the guideline regarding aneurysm diameter threshold for repair in the Netherlands is frequent, 
with a wide variety between vascular surgical teams. Discrepancies between what Dutch vascular surgical teams think they do and they actually do might be an explanation for the frequent and wide variation in guideline deviations. Introducing feedback by clinical auditing might create awareness of occurrence of deviation in VSUs.

\section{ACKNOWLEDGMENTS}

The authors would like to thank all surgeons, registrars, physician assistants, and administrative nurses who registered all the patients in the DSAA, the Dutch Surgical Aneurysm Audit group, and all vascular surgical units that have responded to the survey questionnaire.

\section{REFERENCES}

1. Lo RC, Lu B, Fokkema MT, et al. Relative importance of aneurysm diameter and body size for predicting abdominal aortic aneurysm rupture in men and women. J Vasc Surg. 2014;59:1209-1216.

2. Chaikof EL, Dalman RL, Eskandari MK, et al. The Society for Vascular Surgery practice guidelines on the care of patients with an abdominal aortic aneurysm. J Vasc Surg. 2018;67:2-77. e72.

3. Moll FL, Powell JT, Fraedrich G, et al. Management of abdominal aortic aneurysms clinical practice guidelines of the European society for vascular surgery. Eur J Vasc Endovasc Surg. 2011;41(Suppl 1):S1-S58.

4. Lederle FA, Wilson SE, Johnson GR, et al. Immediate repair compared with surveillance of small abdominal aortic aneurysms. $N$ Engl J Med. 2002;346:1437-1444.

5. Powell JT, Brown LC, Forbes JF, et al. Final 12-year follow-up of surgery versus surveillance in the UK Small Aneurysm Trial. Br J Surg. 2007;94:702-708

6. Ouriel K, Clair DG, Kent KC, et al. Positive impact of endovascular options for treating aneurysms early I. Endovascular repair compared with surveillance for patients with small abdominal aortic aneurysms. J Vasc Surg. 2010;51:1081-1087.

7. Filardo G, Powell JT, Martinez MA, et al. Surgery for small asymptomatic abdominal aortic aneurysms. Cochrane Database Syst Rev. 2015;CD001835.

8. United Kingdom Small Aneurysm Trial Participants, Powell JT, Brady AR, Brown LC, et al. Long-term outcomes of immediate repair compared with surveillance of small abdominal aortic aneurysms. $N$ Engl J Med. 2002;346:1445-1452.

9. Lijftogt N, Vahl AC, Wilschut ED, et al. Adjusted hospital outcomes of abdominal aortic aneurysm surgery reported in the Dutch Surgical Aneurysm Audit. Eur J Vasc Endovasc Surg. 2017;53:520-532.

10. Available at: https://www.dica.nl/jaarrapportage-2014/assets/uploads/DICAJAAR-OFFLINE-2014.pdf. Accessed June 2, 2018.

11. Beck AW, Sedrakyan A, Mao J, et al. Variations in abdominal aortic aneurysm care: a report from the International Consortium of Vascular Registries. Circulation. 2016;134:1948-1958.
12. Zettervall SL, Buck DB, Soden PA, et al. Regional variation exists in patient selection and treatment of abdominal aortic aneurysms. J Vasc Surg. 2016;64:921-927. e921.

13. Karthikesalingam A, Vidal-Diez A, Holt PJ, et al. Thresholds for abdominal aortic aneurysm repair in England and the United States. $N$ Engl J Med. 2016;375:2051-2059.

14. Cao P, De Rango P, Verzini F, et al. Comparison of surveillance versus aortic endografting for small aneurysm repair (CAESAR): results from a randomised trial. Eur J Vasc Endovasc Surg. 2011;41:13-25.

15. Birkmeyer JD, Reames BN, McCulloch $P$, et al. Understanding of regional variation in the use of surgery. Lancet. 2013;382:1121-1129.

16. Tomee SM, Bastiaannet E, Schermerhorn ML, et al. The consequences of real life practice of early abdominal aortic aneurysm repair: a cost-benefit analysis. Eur J Vasc Endovasc Surg. 2017;54:28-33.

17. Darling RC, Messina CR, Brewster DC, et al. Autopsy study of unoperated abdominal aortic aneurysms. The case for early resection. Circulation. 1977;56(3 Suppl):II161-II164.

18. Scott RA, Wilson NM, Ashton HA, et al. Is surgery necessary for abdominal aortic aneurysm less than $6 \mathrm{~cm}$ in diameter? Lancet. 1993;342:1395-1396.

19. Powell JT, Sweeting MJ, Brown LC, et al. Systematic review and metaanalysis of growth rates of small abdominal aortic aneurysms. Br J Surg. 2011;98:609-618.

20. Thompson SG, Brown LC, Sweeting MJ, et al. Systematic review and metaanalysis of the growth and rupture rates of small abdominal aortic aneurysms: implications for surveillance intervals and their cost-effectiveness. Health Technol Assess. 2013;17:1-118.

21. Ho NC, Tran JR, Bektas A. Marfan's syndrome. Lancet. 2005;366:1978-1981.

22. Roberts WC, Honig HS. The spectrum of cardiovascular disease in the Marfan syndrome: a clinico-morphologic study of 18 necropsy patients and comparison to 151 previously reported necropsy patients. Am Heart J. 1982;104:115-135.

23. Hagerty T, Geraghty P, Braverman AC. Abdominal aortic aneurysm in Marfan syndrome. Ann Vasc Surg. 2017;40:294.e1-294.e6.

24. Takayama T, Miyata T, Nagawa $H$. True abdominal aortic aneurysm in Marfan syndrome. J Vasc Surg. 2009;49:1162-1165.

25. Shang EK, Nathan DP, Boonn WW, et al. A modern experience with saccular aortic aneurysms. J Vasc Surg. 2013;57:84-88.

26. Kristmundsson T, Dias N, Resch T, et al. Morphology of small abdominal aortic aneurysms should be considered before continued ultrasound surveillance. Ann Vasc Surg. 2016;31:18-22.

27. Brown LC, Powell JT. Risk factors for aneurysm rupture in patients kept under ultrasound surveillance. UK Small Aneurysm Trial Participants. Ann Surg. 1999;230:289-296 [discussion 296-287].

28. Sweeting MJ, Thompson SG, Brown LC, et al., RESCAN Collaborators. Meta-analysis of individual patient data to examine factors affecting growth and rupture of small abdominal aortic aneurysms. Br J Surg. 2012;99:655-665.

29. Jamtvedt G, Young JM, Kristoffersen DT, et al. Audit and feedback: effects on professional practice and health care outcomes. Cochrane Database Syst Rev. 2006;CD000259. 